\title{
Sustainable Travel and Team Dynamics amongst Mobile Health Professionals
}

\author{
Dr Steve Melia, Senior Lecturer, Centre for Transport and Society \\ University of the West of England
}

\begin{abstract}
This study explores the potential for more sustainable patterns of travel by mobile health professionals. It also explores the relationships between their travel for work and their modal choices in commuting and private travel. It uses as a case study a health trust in the UK which introduced a pioneering scheme, involving the use of electric bikes and pool cars designed to reduce the use of employees' own vehicles for work travel. Using self-categorization theory, it explores the role of work group social norms in explaining the differential take-up of the scheme. The study used an online survey completed by 306 staff, telephone interviews, a focus group, and analysis of carbon emissions. The main alternative to private car use was cycling: by $15 \%$ of staff. Several operational constraints were identified, but the differences in participation were also strongly related to work group cultures. Local managers embodying prototypical norms exerted a significant influence on the work groups which had embraced the scheme. A focus group with a team using electric bikes found evidence of increased staff motivation, and benefits to client groups, due to strengthened in-group social identity. The findings suggest considerable potential for modal shift in the travel of mobile health professionals in urban areas.
\end{abstract}

Keywords: work travel, business travel, health professionals, carbon reductions, mobile staff, cycling, pool cars

\section{Introduction and Aims}

Studies of mobile workers in developed countries have tended to focus on commercial or managerial 'business travellers'. Other types of mobile employee have been largely overlooked. This study focusses on one type of mobile employee which is almost entirely absent from the literature: health care professionals. In a context where most developed countries will be experiencing ageing populations over the coming decades, visits by healthcare professionals to the homes of clients are likely to generate a growing frequency of trips. Most of this travel is currently done by car with a single occupant: the employee. This study explores the potential for more sustainable patterns of travel by mobile health professionals.

The National Health Service in the UK, with 1.3 million employees is the largest employer in Europe (NHS, 2013). Travel by its employees, deliveries and visits to its sites accounts for $5 \%$ of the traffic on the roads in England. The NHS has a carbon reduction strategy which commits it to a cut of $10 \%$ in emissions between 2007 and 2015 (NHS and SDU, 2009). Work travel by staff represented $26 \%$ of the baseline carbon travel emissions for 1990 (NHS et al., 2009). Despite this NHS policies referring to sustainable travel focus on parking, travel to NHS sites by patients and visitors, and commuting by staff, with work travel often overlooked (NHS, 2006).

This study uses as a case study an NHS trust, Avon and Wiltshire Mental Health Trust (AWP), which introduced a pioneering scheme offering electric bicycles and pool cars as alternatives to employees' own cars. The approach was piloted in one small team, which became known as the 'Zero Petrol Team'. Like most teams in the trust, most of the team's work involved visiting clients in their homes. The scheme (which became known as Golow) was then expanded, with the purchase of 20 lowemission diesel Smart cars for use by staff across the trust's sites in Bristol, a city with a population of around 600,000.

Initial discussions with AWP's management revealed that the pattern of use of the pool cars and electric bikes was polarised, with a few parts of the organisation making frequent use of them and many others making little or no use of them. Part of the explanation for this pattern related to operational needs but it was also suggested that some work groups had a different attitude towards 
driving and sustainable transport, for reasons which could be conjectured but had never been investigated.

Prompted by these observations, this study set out with two aims, to explore:

- The operational and personal influences on sustainable work travel amongst AWP's staff

- The influence of, and factors contributing to, work group norms in respect of sustainable work travel

\section{Literature Review}

A search of the international literature revealed several papers about rural medical services in more sparsely populated countries, and some papers about the travel of patients to healthcare facilities, but none about the work travel of healthcare professionals in a comparable context to this study. In a rare example from the peer-reviewed literature referring to the NHS, Cavill et al. (2007) surveyed the directors of public health in English Primary Care Trusts on issues related to cycling. Around a third of the trusts had identified someone with responsibility for cycling - covering the trusts' own staff and/or promotion of cycling in the local population. 37\% paid staff a mileage allowance for cycling on trust business. $8 \%$ provided pool bicycles.

The most comprehensive study of workplace travel plans conducted in the UK included information on the travel plans of $8 \mathrm{NHS}$ trusts or sites (Cairns et al., 2004). It found reductions in driving in 7 out of the 8 cases. As its purpose was to assess the effectiveness of travel plans overall, the published reports (Cairns et al., 2004, 2008) give very limited information about the NHS case studies.

In a comparable context, a few papers have examined the travel practices of social workers involved in home visiting. A central role for the car as "comfort zone, a safe place" emerged unexpectedly from a qualitative study concerned with representations of fear amongst social workers and counsellors (Smith, 2003). Drawing on Sheller's (2004) concepts of "automotive emotions", Ferguson (2009, 2008) outlines a number of other aspects of the relationship between mobile social workers and their cars. Cars are sometimes used as a place of tranquillity, of reflection after difficult encounters with clients, as well as a refuge from "office politics". They sometimes act as a "mobile office", providing confidential space for writing notes, using ICTs, supervision, peer support or debriefing. Cars can provide a "transitional space" which can help to achieve breakthroughs in relationships with clients by placing them in an environment of "encircling warmth", where barriers to communication may be overcome.

Social workers' cars can also be perceived by clients as representations of status and power, particularly as many clients are too poor to own cars themselves. Thus, "while cars bring worker and client together in ever speedier times, the momentum is for cars to drive professionals, so to speak, away from a deeper identification with and connection to their ("car-less") clients" (Ferguson, 2009).

Ferguson (2008) notes that professionals' experience of home visits had not been systematically researched: the observations above are based on limited research evidence. He notes that historically, mobile social work began with the use of bicycles in the late $19^{\text {th }}$ century. As this study will show, bicycles continue to be used by mobile professionals in the NHS. No literature was found on this subject - for health or social work. Apart from passing references in the literature about travel plans, cycling for work purposes (as distinct from cycle commuting) is a little-researched area.

\section{Travel Cultures and Workgroups: Theoretical Perspectives}

In seeking to explain the social context of travel behaviour, a strand in the transport literature emphasises the role of transport cultures, which favour some modes over others. Sheller (2004) emphasises the emotional and sentient forces which influence what are often considered as purely rational decisions about travel behaviour. In another example relevant to this study Aldred and Jungnickel (2012), studied cycling cultures in four British cities including Bristol. They found several examples of workplaces, including the NHS trust used in this case study, where cycling to work had become "normalised". The network of cycle routes offered a "unique way of experiencing the urban environment" with interviewees identifying emotional as well as operational benefits. They also note that clothing exerts an important influence on decisions to cycle. Normal clothing was most common in those places where rates of cycling were highest. 
Many studies have explored the relationship between environmental values and travel behaviour, generally finding it to be weak (e.g. Nilsson and Küller, 2000), although some studies have found that environmental values exert a significant indirect influence via perceived behavioural controls (i.e. how easy or difficult the behaviour seems to the individual) and perceived social norms (Bamberg, 2003, Matthies and Blöbaum, 2007).

Social norms within groups also exert a significant and lasting influence on the personal norms of group members. Several studies have illustrated how the political values of groups such as college sororities influenced members from differing backgrounds, in ways which endured long after the individuals left those environments (Newcomb, 1943 and Seigel and Seigel, 1957 cited in: Hogg and Reid, 2006). Similarly, experimental studies have shown how a group norm, once established, can persist in the group even when the original members have all left (MacNeil \& Sherif, 1976 cited in: Hogg and Reid, 2006).

Amongst the theories used to account for these observations, self-categorization theory explains these influences in terms of social identity. Individuals create or bolster their sense of identity through membership of "in-groups", perceived in contrast to "out-groups" with different characteristics. The process by which social norms evolve within groups is complex and not fully understood. Hogg and Reid (2006) maintain that both observed behaviour and communications within the group help to instil group norms within its members. Conformity to an in-group norm does not necessarily involve copying observed behaviour, however: the individual constructs a perceived in-group norm from observing behaviour within the group and in salient out-groups (Hogg and Abrams, 1988).

In an organisational context, it has been noted that employees tend to identify more strongly with smaller work groups or "sub-units" (Ashforth and Mael, 1989) because "people are more likely to identify with work groups with which they are familiar, and with which they perceive greater similarity. Experimental interventions which increase awareness of the distinctiveness of a sub-group are likely to increase social identification, which may, in turn, increase "engagement and productivity" (van Dick et al., 2005). Within groups, leaders may emerge who embody the prototypical norms. These leaders tend to identify more strongly with the group than others, and to behave in more group-oriented ways. This enables them to generate trust amongst other members, and to lead the group in new directions (Hogg and Reid, 2006).

\section{Methods}

A mixture of quantitative and qualitative methods was used to provide an overview of current practice, to explore travel needs and constraints, and to analyse the reasons for differential take-up of alternatives to the private car. An online survey was designed to ask about work travel, awareness and use of the Golow scheme, with menus of reasons for modal choices and their advantages and problems. These menus included open text 'other' options, explored further in the telephone interviews. The survey also included six statements, designed to assess individual attitudes towards transport and the environment with responses on a 5 point Lickert scale.

A pilot survey was conducted, and an email invitation and reminder were sent to all 1,658 of the trust's staff based in Bristol and South Gloucestershire. Participants in the survey were asked if they would be willing to take part in a second stage of semi-structured telephone interviews, designed to probe issues arising from the survey.

15 respondents were selected for interview on the basis of characteristics relating to specific issues for exploration i.e. works group norms, operational needs and modal shift. Five managers of teams with differing levels of participation in the Golow scheme were interviewed. Amongst the ten other staff, six had reduced or stopped car commuting, and four had stated specific operational constraints on their work travel (e.g. a need to carry equipment or passengers).

A focus group was also conducted with all 7 professional staff working in the 'Zero Petrol Team' at that time, including the team manager. The interview and focus group transcripts were analysed thematically, focussing on operational needs, personal motivations and evidence relating to team dynamics. 


\section{Survey Findings}

The online survey was completed by 306 people, a response rate of $18.5 \%$. Roughly three-quarters were female and two-thirds worked full-time; $18 \%$ worked shifts. Nursing staff and administrative staff both made up around a third of the sample, with other health professionals making up most of the remainder. The frequency of travel for work is shown in Figure 1. Two thirds of the nursing staff travelled at least once a week, compared to $19 \%$ of the administrative staff.

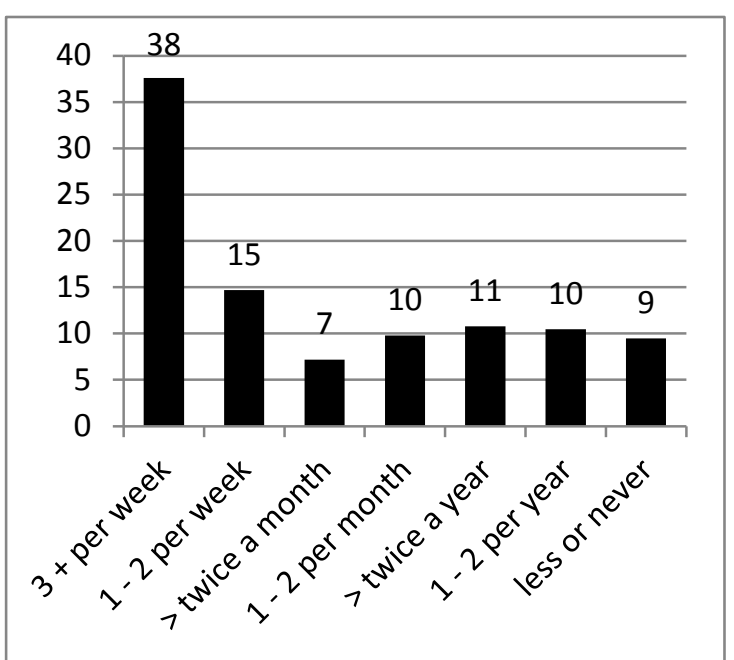

Figure 1 - Frequency of Work Travel (\% of sample)

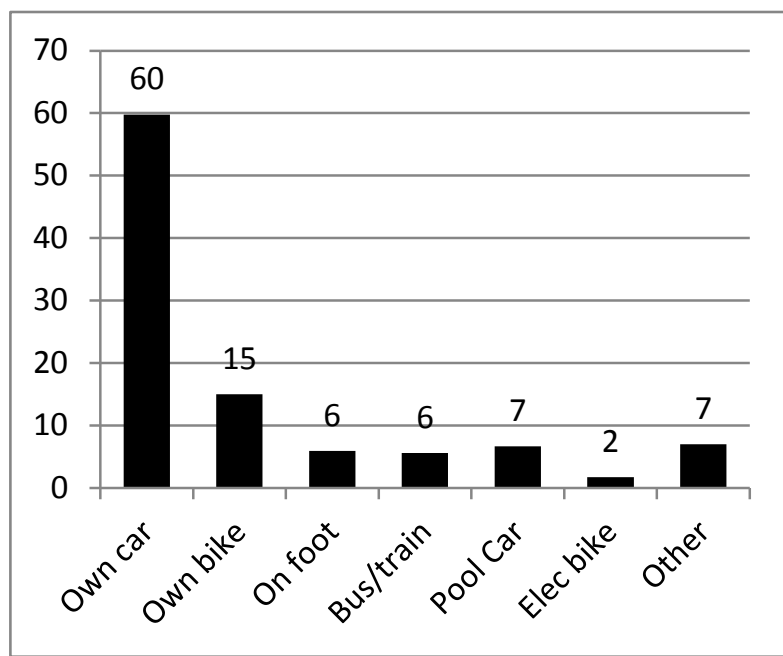

Figure 2 - Main Mode for Work Travel (\% of sample)

As shown on Figure 2, private cars remain the main mode of travel for work, followed by employees' own bicycles.

Two thirds of the nursing staff travelled at least once a week, compared to $19 \%$ of the administrative staff. Household car ownership was similar to the city-wide averages for households containing employees in Bristol from the 2001 Census ( $90 \%$ had at least one). The proportion of car commuters was also very similar to the Census average for Bristol, but more (17\%) cycled and fewer used public transport (18\%) than the Census average.

$72 \%$ of respondents were aware of the Golow scheme, $11 \%$ were unaware and $17 \%$ were "vaguely aware but had not thought about using" the cars or bikes.

Respondents were asked about their reasons for choosing their main mode of travel for work. Table 1 shows the responses for those who mainly drove their own car for work travel:

\begin{tabular}{|l|r|r|}
\hline & No. & $\%$ \\
I often drive straight from home to a client or external site & 64 & 37.4 \\
I feel safer in a car & 44 & 25.7 \\
I prefer driving & 34 & 19.9 \\
I have to carry equipment or heavy files & 31 & 18.1 \\
I sometimes drop off other people on the way & 27 & 15.8 \\
The mileage rate helps towards the running costs of my car & 4 & 2.3 \\
& & \\
Total Asked the Question: & 171 & \\
\hline
\end{tabular}




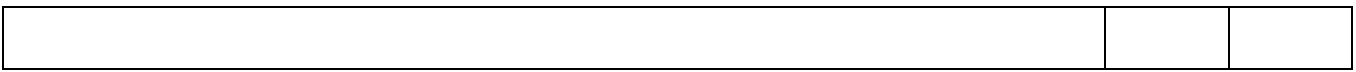

Table 1 - Reasons for Driving for Work (multiple option)

A further 11 respondents (6.4\%) cited other reasons, related to health problems, distance or children.

Table 2 shows the responses from those who cycled, walked or motorcycled to work. This did not include the users of the Golow electric bikes, discussed later.

\begin{tabular}{|l|r|r|}
\hline & No. & $\%$ \\
I feel it is healthier than driving & & \\
I want to make a positive contribution to the environment & 43 & 69.4 \\
It avoids parking problems & 41 & 65.7 \\
I like spending more time in the open air & 36 & 58.1 \\
It is quicker than travelling by car & 32 & 51.6 \\
I only travel short distances for work & 25 & 40.3 \\
I don't own a car & 18 & 29.0 \\
I don't have a driving licence & 17 & 27.4 \\
& 11 & 17.7 \\
Total Asked the Question: & 62 & \\
\hline
\end{tabular}

Table 2 - Reasons for Cycling, Walking or Motorcycling for Work (multiple option)

The most common alternative to driving for work purposes was cycling (15\%). A binary logistic regression was performed with cycling for work as the dependent variable and four covariates: age, gender, frequency of travel and a binary job category (distinguishing the administrative staff and nonclinical managers/professionals, from those directly involved in health or social care). Of these, only gender was significant at the $5 \%$ level. $11 \%$ of women and $23 \%$ of men reported cycling as their main mode. Although the difference was not statistically significant, the over $45 \mathrm{~s}$ in the survey cycled slightly more than their younger colleagues, confounding the expectations of some interviewees who assumed that younger employees were more likely to cycle.

76 respondents $(25 \%)$ had used a pool car at some point: "saving wear on my own car" was the most cited reason for this, by $47 \%$. 27 respondents had used an electric bike at some point. Of those who had not, $12 \%$ stated that they would use one (if available - in some cases no bikes were available to their team); $29 \%$ said 'possibly' and 59\% would not. The reasons for this could equally have applied to cycling in general: $35 \%$ felt safer driving and $22 \%$ found it more convenient.

The survey also included six statements, designed to assess individual attitudes towards transport and the environment. These were used in the statistical analysis described below. One of these statements, shown in Figure 3, enabled a comparison with the British Social Attitude Survey, suggesting that the attitudes of the trust's staff are considerably more pro-environmental than the general population (National Centre for Social Research, 2011). 


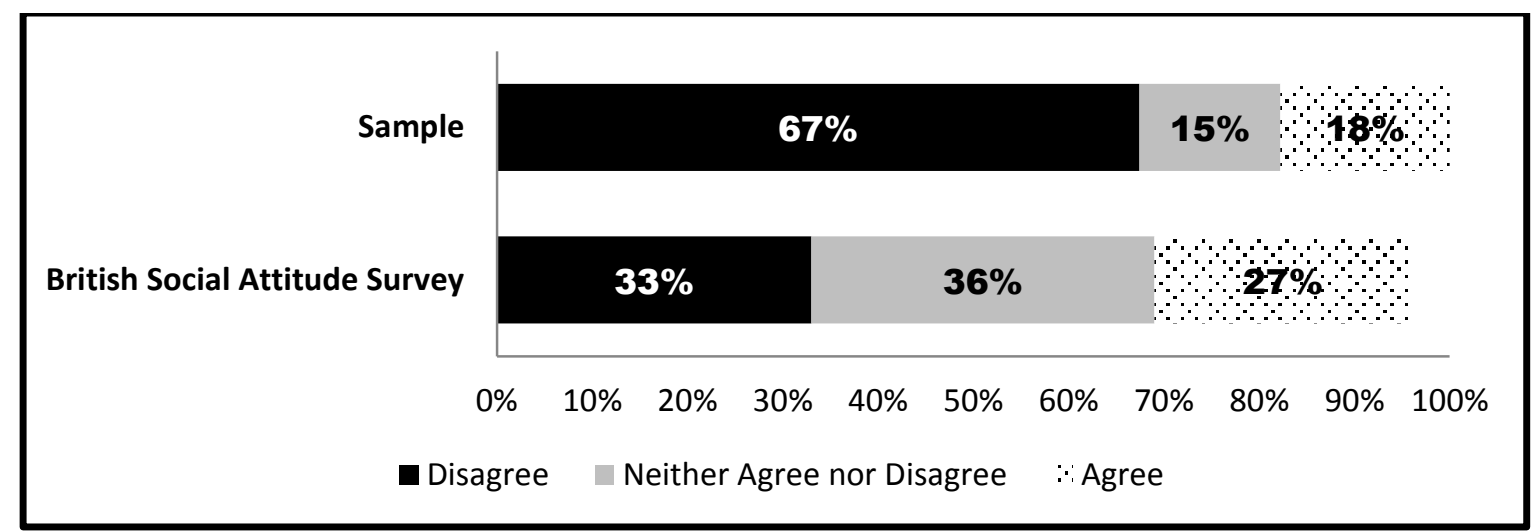

Figure 3 Agreement with the Statement: 'People should be able to use their cars as much as they like, even if it causes damage to the environment'

\section{Work Travel and Operational Constraints}

Most of those interviewed, including the focus group participants, worked in multi-disciplinary community-based teams, with nurses forming the largest proportion. Their travel, like those of the managers, was mainly between fixed bases. Most of the teams covered sectors of the city, so most visits were in relatively compact geographical areas, although several interviewees mentioned that exceptions could occur - where a client moved house for example, and the same team continued to visit them for a transitional period. Some interviewees referred to the unpredictability of their travel needs. This was particularly the case for members of the crisis teams, who could be called at short notice to home visits anywhere within their areas.

The most cited reason for driving for work, by $37 \%$, was the need to travel straight from home to an external site. Several interviewees mentioned that this was their normal pattern. Several of the trust's sites are subject to parking restrictions. Employees commuting by car - and wanting to use it for work travel - are obliged to park their cars some distance from those sites. Eight people mentioned this in the interviews as an advantage of the pool car system. They would commute by other means and use the pool cars when they needed to drive, saving the time that would otherwise have been spent walking to and from private cars parked at a distance.

Carrying equipment and dropping off other people were mentioned as reasons for driving by $18 \%$ and $16 \%$ of those who drove as a main mode, respectively. Most of those interviewed did not need to carry equipment: the few exceptions were relatively small items such as sphygmomanometers. Most interviewees mentioned occasionally carrying other people, usually clients, as passengers.

Those who normally used sustainable modes for work travel were asked whether they occasionally used their own car instead, and if so, under what circumstances. Just under half said they did. The most common circumstance, cited by 19 , was: 'travelling to destinations not reachable by other means'.

Some of those who normally travel for work by sustainable modes used either pool cars or their own cars on occasions where lifts were offered to clients. The need to give lifts varied according to the nature of the clients' health problems. The crisis teams sometimes dealt with clients who would be unable or unlikely to make their own way to appointments. Other staff dealing with less severe cases said they had no need to give lifts.

Some of the issues identified by Ferguson $(2009,2008)$ were raised in some interviews. Two interviewees mentioned using the time spent with clients when driving them. One nurse explained:

"there will be somebody that I've got to take to a hospital appointment... and then we go off somewhere after in the car to talk so we have some privacy, 'cos he's at home with his parents, so there's a couple of times where the use of the vehicle is really important."

Driving and recruitment policy was discussed in interviews with multi-disciplinary team managers and with the human resources department, who explained that the Trust aimed to avoid discrimination against non-drivers in its recruitment policies. A few of the mobile staff neither drove nor cycled using a combination of public transport and walking to reach their appointments. Two of the managers explained that some such employees could be accommodated, providing they remained a small 
minority. If many team members were unable to drive or cycle, this would create operational problems.

\section{Electric Bikes and the Zero Petrol Team}

Of the 27 survey respondents who reported using the electric bikes, $19(70 \%)$ were male, a similar pattern to those who used their own bikes. They included 20 clinical/social work staff, of whom 16 travelled more than three times a week. The main advantages cited in the survey were similar to those using their own bikes: a desire to "make a positive contribution to the environment" $(52 \%)$ and health $(44 \%)$ and "time in the open air" (44\%).

The usual name for the 'Zero Petrol Team' is Bristol Vocational Service. Their function is to help clients obtain or retain employment during or after their treatment. The focus group with the Zero Petrol team focussed on operational needs and team dynamics, both within the team and in its interactions with other parts of the organisation.

There was unanimity in the focus group that the electric bikes were quicker and more reliable than driving. One interviewee explained that commuting by electric bike took around 10 minutes, compared to 20 to 25 minutes by car. A recent experiment conducted by university staff in Bristol provided some objective support for these observations, on another commuting route in Bristol during the rush hour (UWE, 2011).

Greater reliability of timekeeping was cited as one of a range of perceived benefits to clients. Two participants expressed the view that the clients were more appreciative of, or responded better to staff who travelled by bike. One participant suggested that clients were less likely to miss appointments when they knew he would be cycling there.

In a variation on the observations of Ferguson $(2009,2008)$ about car use by social workers, two participants mentioned that they had cycled with clients and that this activity helped the process of recovery and motivation which is important for helping mental health clients to obtain or retain employment.

These impressions about advantages for clients were linked to others about advantages for staff, particularly related to health, fitness, mood and motivation. The "de-stressing" properties of cycling, particularly on the off-road paths were contrasted with the stress of driving in heavy traffic. The link between the states of mind of staff and clients was believed to be particularly important in a unit involved in support and recovery:

"We are trying to inspire people to move their lives forward and this all adds into it, you can't separate that. We are much more invigorated in our work, and that is partly because of this ... bike thing."

In a reflection of Sheller (2004)'s observations on automotive emotions, there was evidence of emotional attachment to the bikes themselves. These were expressed explicitly by the three female participants who all said: "we love our bikes". Two participants were social workers, employed by Bristol City Council, who had worked as part of the trust's team, but were about to be separated in a reorganisation. There was some uncertainty over whether they would be able to keep their electric bikes, and both of them made a strong plea to be able to keep them. Apart from the operational problems losing the bikes would cause, the demeanour of at least one participant suggested a possible emotional loss.

One factor promoting a degree of emotional attachment - as well as administrative simplicity - is that, unlike the pool cars, each electric bike in the Zero Petrol Team was allocated to one person, who would ride it home at the end of the day, with no booking involved. One focus group participant commented that being able to ride the bike home was "what makes it work". In other locations where electric pool bikes were made available, these were either used frequently by one person at a time, or else, were used only infrequently.

Bristol's status as a cycling city was mentioned several times in the focus group, with one participant explaining that cycling is "not seen as bizarre" in Bristol, although the attitudes of the Zero Petrol Team were contrasted with those of some other teams, where cycling was less common. 


\section{Attitudes and Factors Influencing Participation}

The influence of operational and team-related factors on participation in the Golow scheme was investigated in two ways: through statistical analysis of the survey responses, and through the interviews and focus group. The survey included six measures of individual attitudes towards transport and the environment, as well as questions on travel behaviour, demographic information and the attitudes of colleagues and management towards the Golow scheme. A binary logistic regression was performed with use of the pool car as the dependent variable. The covariates included the six measures of individual attitude as well as the following eight binary variables:

- Frequent traveller (> 3 times per week for work)

- Management positive towards the Golow scheme,

- Colleagues positive towards the Golow scheme

- Car in the household

- Age (over/under 45)

- Job (clinical/support - the former includes a small number of social workers)

- Gender

- Usually commutes by own car

\begin{tabular}{|c|c|c|c|c|c|c|c|}
\hline & & $\mathrm{B}$ & S.E. & Wald & df & $\rho$ & Odds Ratio \\
\hline \multirow[t]{5}{*}{ Step $4^{a}$} & Frequent traveller & 1.047 & .345 & 9.230 & 1 & .002 & 2.848 \\
\hline & Management positive & 1.378 & .361 & 14.618 & 1 & .000 & 3.969 \\
\hline & Colleagues positive & .837 & .352 & 5.656 & 1 & .017 & 2.310 \\
\hline & Support job & -.982 & .394 & 6.205 & 1 & .013 & .375 \\
\hline & Constant & -2.130 & .331 & 41.471 & 1 & .000 & .119 \\
\hline
\end{tabular}

a. Variable(s) entered on step 4: Colleagues positive

Table 3 - Binary Logistic Regression on Probability of Using a Pool Car ( $n=306)$

The variables included in the resulting model are shown in Table 3 . Interestingly, none of the individual attitudes improved the fit of the model, whereas the perceived attitudes of management and colleagues were both significant: those perceiving a positive attitude amongst their management were nearly four times (3.969) as likely to use a pool car as those whose managers are sceptical, or unaware. These issues were explored further in the interviews. As expected, clinical staff and more frequent travellers were more likely to use the pool cars (those who never travelled for work were excluded from the dataset).

Although none of the individual attitudes were significant in the regression model, a bivariate crosstabulation revealed a negative association between pool car use and agreement with the statement: "people should be allowed to use their cars as much as they like, even if it causes damage to the environment" $\left(X^{2}=4.361, \mathrm{df}=1, \rho=0.037\right)$. This is consistent with the indirect effect of environmental attitudes found by Bamberg (2003) and Matthies and Ellen (2007). Further crosstabulations revealed strong associations between the same measure of environmental attitudes and two of the variables included in the regression model: the perceptions of management and colleagues' attitudes towards the Golow scheme.

There are two plausible explanations for these associations, which are not mutually exclusive. The first is the 'false consensus effect' whereby people tend to "see their own behavioural choices and judgements as relatively common and appropriate to existing circumstances" (Ross et al., 1977). The mechanisms by which this occurs are many and complex, but a substantial body of evidence suggests that people's perceptions are often influenced in this way (Mullen et al., 1985). So in this 
case, the environmental values would influence more specific attitudes towards the Golow scheme, and this in turn would influence how individuals perceived the attitudes of others towards the scheme.

The second explanation, which could be partly influenced by the first, relates to group norms (Hogg and Reid, 2006). These issues were probed in the interviews and focus group, discussed below.

A similar analysis was performed for use of the electric bikes. A binary logistic regression was performed with covariates including the six measures of individual attitude and the first 7 of the 8 binary variables shown in Table 3 plus: "cyclist outside work".

\begin{tabular}{|c|c|c|c|c|c|c|c|}
\hline & & $\mathrm{B}$ & S.E. & Wald & df & $\rho$ & Odds Ratio \\
\hline \multirow[t]{6}{*}{ Step $5^{\mathrm{a}}$} & Management positive & 1.421 & .636 & 4.988 & 1 & .026 & 4.140 \\
\hline & Colleagues positive & 1.447 & .625 & 5.360 & 1 & .021 & 4.251 \\
\hline & No car in household & 1.505 & .739 & 4.143 & 1 & .042 & 4.505 \\
\hline & Cyclist outside of work & 1.651 & .637 & 6.714 & 1 & .010 & 5.215 \\
\hline & Gender (male) & 2.176 & .569 & 14.640 & 1 & .000 & 8.811 \\
\hline & Constant & -6.168 & .886 & 48.473 & 1 & .000 & .002 \\
\hline
\end{tabular}

Table 4 Binary Logistic Regression on Probability of Using an Electric Bike $(n=306)$

The variables included in the model are shown in Table 4. As expected, men were more likely to use the electric bikes as were people with no car and those who cycled outside of work. The Zero Petrol Group was an exception to this pattern - all team members used the electric bikes, regardless of gender. Some of the female members had taken up cycling (or 'utility cycling' for one woman who cycled for leisure) for the first time as adults as a result of the scheme.

As with the pool cars, the perceived attitudes of management and colleagues improved the fit of the model in Table 4, whereas the measures of individual attitude were significant in a binary crosstabulation $\left(\mathrm{X}^{2}=4.490, \mathrm{df}=1, \rho=0.034\right)$ but not in the regression model. This suggests some type of indirect relationship between environmental attitudes and electric bike use.

Some interviewees commented on the differences in participation rates of different teams. One manager was responsible for two teams, one of which made considerable use of pool cars and electric bikes. The other team made less use of both. In seeking to explain the difference, he described the low-use team as more of a "traditional team" - they were slightly older on average; more of them lived outside Bristol and commuted by car. Both areas were urban and compact, but the high users covered a more central area of the city. He also explained that the organisation of caseloads differed between the two. In the low user group, clients were allocated to individual staff, whereas the high user group shared their caseloads, "so there's more of a... philosophy of sharing things..."

As suggested by Taylor (2007), there was evidence of some local managers, often motivated by environmental considerations, providing leadership on these issues, particularly in the focus group with the Zero Petrol Team, where the Golow manager had originally been the team manager, playing the role of the leader embodying 'prototypical norms' discussed by Hogg and Reid (2006).

Several participants mentioned varying levels of environmental commitment. One expressed strong personal convictions. Another explained that former team manager (who became the Golow manager) had been motivated by a "green agenda", which had influenced the team. 
There was considerable evidence that the use of electric bikes had strengthened in-group norms and the sense of group identity, partly by reference to salient outgroups, as posited by Hogg and Abrams (1988). For example:

"It was part of the identity of the service you provide, identified as that, that's why none of us would like to go back to our cars now, because that would feel like letting the team down."

When the team was first established, the electric bikes generated interest in the media, involving the team with the Chief Executive. There was initially some concern about possible resentment amongst other the teams with whom they were to be "embedded". These factors appeared to strengthen the group's perception of other distinguishing characteristics - the nature of their job being rather different from the teams with which they worked. As the team became more established, this perception declined. Nowadays, several participants added, the use of electric bikes was accepted as just: "something that we do, part of our job".

There was a consensus that the electric bikes had brought the team together in other ways increasing their motivation (in the ways described by van Dick et al., 2005) and their socialising outside of work.

Group social norms may persist, even when group membership changes. The way in which new members are inducted into the group is important to this process. The manager explained how she approached the issue with new recruits or temporary assignees:

"It's done in a very matter of fact way. It's like: we are all on bikes and this is the way we operate. We have got one for you; here is the equipment; is it going to be a problem? Do you want a course, a safety course?... if there are health grounds or whatever, I am happy to talk about that but actually the whole team attitude, including myself, is: this is the way it is..."

\section{Conclusions}

This study set out to explore the potential for more sustainable travel amongst a hitherto littleresearched group: mobile health professionals. Many of the findings would be equally applicable to other groups of mobile workers required to visit people in their homes in urban areas. It found considerable use and considerable potential for work travel by sustainable modes in a context where many employers assume that car travel is the only feasible alternative.

The most common alternative to driving - both for commuting and for work travel - was cycling, using the employee's own bike in most cases, although electric bikes offer significant benefits which could be more widely exploited. A small minority of employees neither cycled nor drove and were able to fulfil the requirements of the job by walking and public transport. For operational reasons, any strategy to reduce private car use would need to largely rely on pool cars and cycling, with particular benefits from electric bikes. Public transport is appropriate for some longer and irregular journeys (e.g. to meetings) but would be less relevant for the daily travel of community-based staff.

Many of the staff who mostly travelled by sustainable modes reported occasional needs for a car, when travelling outside their normal area, or carrying clients as passengers, for example. Although some of these people have a private car available, for others pool cars are essential - unless those journeys are allocated to another member of staff. The availability of pool cars has enabled many employees ( $30 \%$ of users in the survey) to reduce their commuting by car and in some circumstances, their household car ownership.

Team social norms were an important factor in the acceptance and take-up of the Golow scheme and alternatives to private car use in general. Perceptions of attitudes amongst colleagues and managers were both significantly associated with participation, whereas measures of individual attitude were not. Although it is a small-scale example, the experience of the Zero Petrol Team suggests that subgroups may create new social norms which differ from those of the wider society and also provide practical support - in this case, to help female team members take up cycling for work. The prototypical role of local managers in this context may have broader implications for senior managers seeking to foster change in workplace cultures. Those local managers whose values and personal behaviour support the cultural change have in this example exerted a powerful influence on their work teams. 
Although the Golow scheme represents a fairly small proportion of the travel in the case study trust, the findings of this this study suggest that the scope for this type of change is considerable. Only $31 \%$ of non-users said they would not consider using one of the pool cars. $59 \%$ of non-users said they would not consider using an electric bike. For most of these people, the reasons related to personal preferences, influenced by work group norms, rather than the nature of the travel itself. For those people who are not able or willing to cycle, a mixture of alternatives can still help to reduce private car use, where this is an agreed objective.

A substantial literature exists on transport behaviour change in general and specific guidance has been published for NHS trusts in the UK (NHS, 2006, NHS SDU, 2009). The findings of this study would reinforce some of those recommendations (e.g. the need to understand current transport patterns, and provide supportive facilities and equipment) and would add that organisations wanting to reduce emissions and promote more active travel should: encourage local managers to set team transport norms, where travel by alternatives to driving become 'normalised'. This may begin on a 'bottom up' basis, but senior managers may also help to set an example (and avoid perceptions of double standards). They should also ensure that recruitment and administrative procedures do not send out the message that driving is the normal option, as many other organisations, regularly do. When introducing a new programme or change in practices, the values of individual managers should be taken into account when selecting teams or departments to pilot, or begin a new programme.

This study has suggested that occasional car use is important for most of the people who normally travel by other means, so the provision of pool cars should be seen as one element in a strategy to promote cycling and walking for work purposes - and to reduce commuting by car.

The findings also suggest that parking restrictions can be a powerful motivator for modal shift within urban areas. Where parking is addressed in local transport planning, the focus is usually on commuters, shoppers or the influence of residential parking on household travel behaviour. This study suggests that parking controls may also reduce work-related traffic in inner city areas.

\section{References:}

Aldred, R. and Jungnickel, K., (2012) Cycling Cultures, Summary of Key Findings and Recommendations [online]. Report number: June.http://cyclingcultures.org.uk/outputs-papers.html: University of East London;ESRC. [Accessed June 28th 2012]

Ashforth, B.E. and Mael, F. (1989) Social identity theory and the organization. Academy of Management Review. 14 (1), pp. 20-39.

Bamberg, S. (2003) How does environmental concern influence specific environmentally related behaviors? A new answer to an old question. Journal of Environmental Psychology. 23 (1), pp. 21-32.

Cairns, S., Sloman, L., Newson, C., Anable, J., Kirkbride, A. and Goodwin, P. (2008) Smarter Choices: Assessing the Potential to Achieve Traffic Reduction Using 'Soft Measures'. Transport Reviews: A Transnational Transdisciplinary Journal. 28 (5), pp. 593.

Cairns, S., Sloman, L., Newson, C., Anable, J., Kirkbride, A. and Goodwin, P., (2004) Smarter Choices - Changing the Way we Travel: Main Document. London: Department for Transport.

Cavill, N., Rutter, H. and Hill, A. (2007) Action on cycling in primary care trusts: Results of a survey of Directors of Public Health. Public Health. 121 (2), pp. 100-105.

Ferguson, H. (2009) Driven to care: the car, automobility and social work. Mobilities. 4 (2), pp. 275293. 
Ferguson, H. (2008) Liquid social work: Welfare interventions as mobile practices. British Journal of Social Work. 38 (3), pp. 561.

Hogg, M.A. and Abrams, D. (1988) Social Identifications: A Social Psychology of Intergroup Relations and Group Processes. London and New York: Psychology Press.

Hogg, M.A. and Reid, S.A. (2006) Social Identity, Self-Categorization, and the Communication of Group Norms. Communication Theory. 16 (1), pp. 7-30.

Matthies, E. and Blöbaum, A. (2007) Ecological Norm Orientation And Private Car Use. In: Gärling, T. and Steg, L., eds. (2007) Threats from Car Traffic to the Quality of Urban Life: Problems, Causes, and Solutions. Oxford: Elsevier, pp. 251-274.

Mullen, B., Atkins, J.L., Champion, D.S., Edwards, C., Hardy, D., Story, J.E. and Vanderklok, M. (1985) The false consensus effect: A meta-analysis of 115 hypothesis tests. Journal of Experimental Social Psychology. 21 (3), pp. 262-283.

National Centre for Social Research, (2011) British Social Attitudes Survey. Report number: 28.London: Sage.

NHS (2013) Not Sure? - NHS Careers Available from: http://www.nhscareers.nhs.uk/explore-bycareer/not-sure/ [Accessed 5/7/2013].

\section{NHS, (2006) Health Technical Memorandum 07-03 Transport Management and Car-Parking. London: TSO.}

NHS SDU, (2009) Low Carbon Travel, Transport, and Access [online]. Report number: January.http://www.sdu.nhs.uk/documents/publications/1232901538 QFPD low carbon travel, tran sport and access.pdf: NHS Sustainable Development Unit. [Accessed June 2012].

NHS, Arup and SEI, (2009) NHS England Carbon Emissions: Carbon Footprint Modelling to 2020 [online]. Report number: January.http://www.sdu.nhs.uk: NHS Sustainable Development Unit; Arup ;Stockholm Environment Institute. [Accessed June 2012].

NHS and SDU (2009) Saving Carbon, Improving Health [online]. Cambridge: NHS Sustainable Development Unit.

Nilsson, M. and Küller, R. (2000) Travel behaviour and environmental concern. Transportation Research Part D: Transport and Environment. 5 (3), pp. 211-234.

Ross, L., Greene, D. and House, P. (1977) The "false consensus effect": An egocentric bias in social perception and attribution processes. Journal of Experimental Social Psychology. 13 (3), pp. 279-301.

Sheller, M. (2004) Automotive Emotions: Feeling the Car. Theory, Culture \& Society. 21 (4), pp. 221242.

Smith, M. (2003) Gorgons, cars and the frightful fiend: Representations of fear in social work and counselling. Journal of Social Work Practice: Psychotherapeutic Approaches in Health, Welfare and the Community. 17 (2), pp. 153-162. [Accessed June 15, 2011].

Taylor, I., (2007) The Essential Guide to Travel Planning. [online].

http://www.orsa.org.uk/info/travel planning guide.pdf: Department for Transport; National Busoness Travel Network. [Accessed June 14th 2012].

UWE (2011) Bristol UWE Travel Challenge. Available from:

http://info.uwe.ac.uk/news/uwenews/news.aspx?id=2099 [Accessed 7/5/2012]. 
van Dick, R., Wagner, U., Stellmacher, J., Christ, O. and Tissington, P.A. (2005) To Be(long) or Not to $\mathrm{Be}$ (long): Social Identification in Organizational Contexts. Genetic, Social, and General Psychology Monographs. 131 (3), pp. 189-218. 